\title{
Effects of minodronate in postmenopausal women with osteoporosis who received prior treatment with raloxifene
}

This article was published in the following Dove Press journal:

International Journal of Women's Health

13 November 2017

Number of times this article has been viewed

\author{
Aska Toda \\ Kenjiro Sawada \\ Akihiko Yoshimura \\ Erika Nakatsuka \\ Hiromasa Kuroda \\ Katsumi Kozasa \\ Mayuko Miyamoto \\ Kae Hashimoto \\ Seiji Mabuchi \\ Tadashi Kimura \\ Department of Obstetrics and \\ Gynecology, Osaka University \\ Graduate School of Medicine, \\ Suita, Osaka, Japan
}

Correspondence: Kenjiro Sawada Department of Obstetrics and Gynecology, Osaka University Graduate School of Medicine, 2-2 Yamadaoka, Suita, Osaka 565-087I, Japan

$\mathrm{Tel}+8 \mathrm{I} 66879335 \mathrm{I}$

Fax +8I 668793359

Email daasawada@gyne.med.osaka-u.ac.jp
Background: In clinical practice, patients with postmenopausal osteoporosis have often shown a poor response to treatment with an antiresorptive agent for several years. The purpose of this study was to investigate the efficacy of switching raloxifene with minodronate in patients who responded poorly to the treatment of postmenopausal osteoporosis with raloxifene.

Patients and methods: This observational study was conducted based on a single-arm, non-randomized, open-label design and was approved by the institute's institutional review board. Postmenopausal women with osteoporosis who became unresponsive in terms of bone mineral density (BMD) after being administered raloxifene for two or more years were enrolled. Patients were treated with $1 \mathrm{mg}$ minodronate daily or $50 \mathrm{mg}$ minodronate monthly. Changes in BMD and serum bone turnover markers were monitored at baseline, 6, 12, and 24 months after switching treatment.

Results: Twenty-seven patients were enrolled. Two discontinued treatment because of adverse events related to the study drug. Among the remaining 25 patients, lumbar BMD significantly increased by $3.67 \%, 5.08 \%$, and $6.97 \%$ at 6,12 , and 24 months, respectively, and femoral neck BMD increased by $1.63 \%, 2.18 \%$, and $3.85 \%$ at 6,12 , and 24 months, respectively. Serum bone-specific alkaline phosphatase showed a significant reduction of $30.35 \%$ from the baseline $(p<0.0001)$ within the first 6 months, suggesting a stronger antiresorptive effect of minodronate. Serum N-terminal telopeptide of type I collagen showed a tendency to decrease.

Conclusion: Switching raloxifene with minodronate is effective in poor responders of osteoporosis treatment and should be considered as one of the treatment options for osteoporosis.

Keywords: osteoporosis, minodronate, raloxifene, switching therapy

\section{Introduction}

Postmenopausal osteoporosis is the most common bone disease and is related to a low bone mineral density (BMD) and pathological fractures, which lead to significant morbidity. ${ }^{1}$ In addition, the risk of osteoporotic fracture associated with surgical menopause has proven to be increased compared with that in women who have intact ovaries, even in elderly women after menopause. ${ }^{2}$ These osteoporotic fractures and pain caused by postmenopausal estrogen deficiency have a long-term serious influence on health-related quality of life. In a rapidly increasing aged population worldwide, a reduction of the burden of osteoporosis in the coming years is clearly needed.

Therapeutic treatments recommended for osteoporosis include bisphosphonates (alendronate [ALN], risedronate [RIS], minodronate [MIN]), selective estrogen-receptor modulators (raloxifene [RAL], bazedoxifene), a full human monoclonal antibody to RANKL (denosumab), active vitamin $\mathrm{D}_{3}$ derivatives (alfacalcidol, eldecalcitol), and 
recombinant parathyroid hormone (teriparatide). ${ }^{3}$ Of these, RAL, a nonsteroidal benzothiophene derivative of the selective estrogen receptor-modulator class, has been shown to reduce the relative risk of vertebral fractures by up to $69 \%$ in postmenopausal Caucasian women with osteoporosis after 3 years of treatment, compared with placebo. ${ }^{4}$ However, in clinical practice, it has often been reported that some women with postmenopausal osteoporosis have a decreased BMD while taking RAL constantly. Indeed, in a largescale postmarketing surveillance of RAL in Japan, lumbar BMD increased significantly compared with baseline at $6,12,24$, and 36 months $(2.51 \%, 2.85 \%, 4.76 \%$, and $3.51 \%$, respectively). ${ }^{5}$ This study demonstrated that a considerable number of patients experienced decreased BMDs after 2-years of treatment with RAL; however, few studies have reported on the timing on when to switch treatment drugs. MIN is an oral nitrogen-containing bisphosphonate developed in Japan, which has a stronger inhibitory effect on farnesyl pyrophosphate synthase in osteoclasts, compared with other bisphosphonates. ${ }^{6}$ With regard to bone resorption inhibition, MIN is 1,000 times more effective than etidronate and 10-100 times more effective than ALN. ${ }^{7}$ A randomized placebo-controlled trial showed that MIN treatment reduced vertebral fractures by $59 \%$ without serious adverse events in Japanese postmenopausal women. ${ }^{8}$

With these in mind, we aimed to analyze the effects of MIN on BMD and bone turnover markers in postmenopausal women with osteoporosis who had received prior treatment with RAL and to investigate the validity of switching from RAL to MIN.

\section{Patients and methods \\ Patients and study design}

This observational study was conducted based on a singlearm, non-randomized, open-label design. The women in this study were naturally or surgically menopausal ( $>12$ months since the last menstrual period or after oophorectomy). All were Japanese, aged 36-77 years and from a middle-class community in the northern part of Osaka prefecture in Japan. Lumbar (at the L2-L4 vertebral site) and femoral BMDs were measured by dual-energy X-ray absorptiometry (DXA) (Discovery-A, Hologic, Bedford, MA, USA). The coefficient of variance was $<1.0 \%$ for the lumbar spine in our institution. Based on their BMDs, patients with osteoporosis (below -2.5 SD of the mean value of healthy 20-44-yearold Japanese women) were diagnosed, and $60 \mathrm{mg}$ RAL (Lilly Co., Ltd., Indianapolis, IN, USA) was prescribed as prior treatment. After at least a 2-year treatment with RAL, patients whose BMD did not increase, or whose BMD increased initially but who became unresponsive after RAL administration were asked to participate in this study. This study was conducted in accordance with the ethical standards of the Helsinki II Declaration, was approved by the institutional review boards at Academic Clinical Research Center of Osaka University, and registered as a clinical study in UMIN (UMIN000013916). Patients were excluded for the following reasons: 1) concomitant use of other agents for osteoporosis, 2) concomitant use of other agents that potentially affect bone turnover, 3 ) presence of esophageal stricture or achalasia, 4) inability to remain upright for $30 \mathrm{~min}$ after dosing, 5) hypersensitivity to MIN or other bisphosphonate, 6) hypocalcemia, 7) aphagia, esophagitis, gastritis, duodenitis, or ulcers, 8) severe nephropathy, and 9) inappropriate to participate in the study. Participant recruitment was done between September 2010 and February 2015.

After written informed consent were provided, the patients were treated with $1 \mathrm{mg}$ MIN (Astellas Pharma Inc., Tokyo, Japan) once a day or $50 \mathrm{mg}$ MIN (Astellas Pharma Inc.) once a month for 24 months. Participants were instructed to take the tablets with $200 \mathrm{~mL}$ water at least $30 \mathrm{~min}$ before breakfast and not to lie down within 30 min after taking the medication. Other supplemental osteoporosis treatments, such as active vitamin D and calcium, were continued during the study period. Adverse events were recorded at all post and baseline visits, and their severity and relationship to treatment were evaluated by the investigators.

\section{BMD measurement}

BMDs of the lumbar spine (L2-L4) in the posteroanterior projections and of the femoral neck were measured by dualenergy X-ray absorptiometry (Discovery-A) at baseline, 6,12 , and 24 months after the treatment.

\section{Biochemical bone markers}

At baseline and at 6, 12, and 24 months, the bone absorptive parameter, serum N collagen-type $N$-telopeptide (NTX), was measured by enzyme-linked immunosorbent assay (SRL [Tokyo, Japan]; normal range for women: 7.5-16.5 nmol BCE/L [ $\leq 45$ years], 10.7-24.0 nmol BCE/L [ $>45$ years]). The bone formation marker, serum bone-specific alkaline phosphatase (BAP), was measured by enzyme immunoassay (SRL; normal range: 9.6-35.4 U/L) until March 2011, and chemiluminescent enzyme immunoassay (SRL; normal range for women: $2.9-14.5 \mu \mathrm{g} / \mathrm{L}[\leq 45$ years] $3.8-22.6 \mu \mathrm{g} / \mathrm{L}$ [ $>45$ years] ) since April 2011. BAP values were converted based on the compensation formula supplied by the manufacturer. 


\section{Statistical analysis}

Paired Student's $t$-test was used to make baseline comparisons of the BMD and bone turnover markers. Results were considered significant at $p<0.05$. All analyses were performed using JMP Pro 10 (SAS Institute Inc., Cary, NC, USA).

\section{Results}

\section{Patient characteristics}

This study enrolled 27 subjects. Baseline characteristics are summarized in Table 1. The median age of the participants was 66 years (range, 37-77 years), and the mean body mass index was $20.3 \pm 3.0 \mathrm{~kg} / \mathrm{m}^{2}$. Four patients $(14.8 \%)$ were previously treated with other bisphosphonates (etidronate $[\mathrm{n}=2]$, ALN [n=2]) before RAL treatment. Two patients (7.4\%) discontinued treatment because of adverse events related to the study drug (one experienced epigastric distress at one month, and the other experienced anorexia at four months), and 25 patients completed 24 months of treatment. During the study period, no patient experienced new clinical fractures.

\section{BMD}

Switching RAL to MIN significantly increased lumbar BMD rapidly during the first 6 months $(3.67 \% \pm 3.32 \%)$ and gradually increased thereafter $(5.08 \% \pm 3.81 \%$ at 12 months, and $6.97 \% \pm 4.12 \%$ at 24 months), whereas it decreased by $2.61 \% \pm 2.32 \%$ on prior treatment with RAL after 12 months (Figure 1A). Switching RAL to MIN similarly increased femoral neck BMD by $1.63 \% \pm 3.25 \%, 2.18 \% \pm 6.19 \%$, $3.85 \% \pm 5.96 \%$ at 6,12 , and 24 months, respectively, whereas

Table I Demographics and baseline characteristics of the study population

\begin{tabular}{ll}
\hline Characteristics & Values \\
\hline Age $($ years $)$, median (range) & $66(36-77)$ \\
Height $(\mathrm{cm})$ & $154.7 \pm 5.6$ \\
Weight $(\mathrm{kg})$ & $48.5 \pm 7.0$ \\
Body mass index $\left(\mathrm{kg} / \mathrm{m}^{2}\right)$ & $20.3 \pm 3.0$ \\
Lumbar BMD $\left(\mathrm{g} / \mathrm{cm}^{2}\right)$ & $0.719 \pm 0.1 \mathrm{I} 3$ \\
Femoral neck BMD $\left(\mathrm{g} / \mathrm{cm}^{2}\right)$ & $0.543 \pm 0.09$ I \\
Serum NTX $(\mathrm{nmol}$ BCE/L) & $15.44 \pm 4.93$ \\
Serum BAP $(\mathrm{U} / \mathrm{L})$ & $13.02 \pm 3.46$ \\
Previous HRT, $\mathrm{n}(\%)$ & $5(18.5)$ \\
Previous bisphosphonate use, $\mathrm{n}(\%)$ & $4(14.8)$ \\
Etidronate & $2(7.4)$ \\
Alendronate & $2(7.4)$ \\
Risedronate & $0(0.0)$ \\
\hline
\end{tabular}

Note: Data are expressed as mean $\pm S D$, unless otherwise noted.

Abbreviations: BMD, bone mineral density; NTX, N collagen-type $\mathrm{N}$-telopeptide; BAP, bone-specific alkaline phosphatase; HRT, hormone replacement therapy; BCE, bone collagen equivalent. it decreased by $1.27 \% \pm 3.47 \%$ during 12 months of pretreatment with RAL (Figure 1B).

\section{Bone turnover marker}

Percentage change in bone turnover markers from the baseline are shown in Figure 2. Switching RAL to MIN induced a reduction of serum NTX by $12.95 \% \pm 21.60 \%$ from baseline $(p=0.0305)$ at 6 months; however, no significant changes were seen after 12 and 24 months (Figure 2A). For serum BAP, it caused a significant reduction of $30.35 \% \pm 16.92 \%$, $33.56 \% \pm 20.72 \%$, and $28.01 \% \pm 27.20 \%$ from the baseline at $6,12(p<0.0001)$ and 24 months $(p=0.0016)$, respectively (Figure 2B). In contrast, prior treatment with RAL did not significantly affect serum NTX and BAP values.

\section{Discussion}

RAL, which has been used to treat postmenopausal osteoporosis in Japan since May 2004 (60 mg tablets), has been shown to increase lumbar spine BMD, improve bone strength, and prevent vertebral fractures, as well as reduce the risk of invasive breast cancers. ${ }^{9-11}$ Thus, RAL remains one of the first treatment options for postmenopausal osteoporosis. However, we often encounter women with postmenopausal osteoporosis whose BMDs decrease despite the constant use of RAL. In the present study, we report that switching RAL to MIN in these patients significantly increased their lumbar and femoral BMDs, accompanied by a significant decrease of bone turnover marker, serum BAP. Particularly, lumbar spine BMD significantly increased by $5.08 \% \pm 3.81 \%$ and $6.97 \% \pm 4.12 \%$ at 12 and 24 months, respectively, which showed a comparable effect as a primary treatment by MIN administration for postmenopausal osteoporosis in a large prospective study (6.1\% at 12 months). ${ }^{12}$

It remains a clinical issue whether the treatment drug should be altered in women with osteoporosis treated longterm with antiresorptive agents, such as bisphosphonates (ALN, RIS, or MIN) or selective estrogen-receptor modulators (bazedoxifene, RAL) when they are not responding to these agents. Although RAL is known to continuously exert the preventive effect against vertebral fractures for at least 3 years, ${ }^{9}$ decreased BMD might lead to poor compliance from patients in clinical practice if they are informed about this information. Thus, the protocol of alternative treatment options would be meaningful to have and should be prepared; however, no protocols have conclusively been determined. Although several studies regarding switching therapies have been reported, many of them are focusing on teriparatide rather than antiresorptive agents because it is 
A

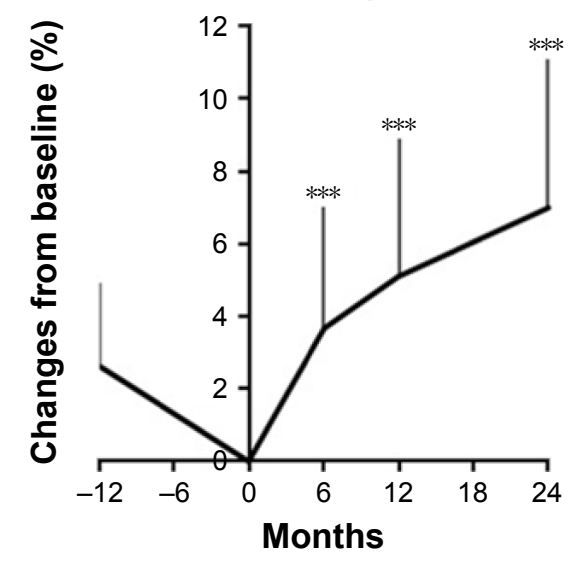

B

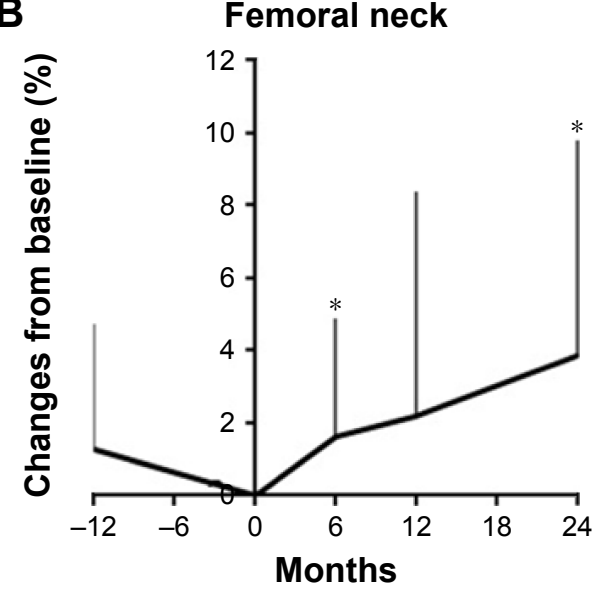

Figure I Percentage change of BMD of the lumbar spine (L2-4) (A) and femoral neck (B) from the baseline during the follow-up period. Notes: Points: mean, vertical lines: standard deviation, ${ }^{*} p<0.05$ and ${ }^{* * *} p<0.00$ I versus baseline.

Abbreviation: BMD, bone mineral density.

the only anabolic agent available and indicated for the treatment of men and postmenopausal women with osteoporosis who are at high risk for fracture, including those who have failed or are intolerant of previous osteoporosis therapy. ${ }^{13-15}$ However, teriparatide is usually used as a second-line treatment for patients with severe osteoporosis who are at high risk for fracture or have osteoporotic fractures and is therefore, not indicated for every patient. ${ }^{16}$ Eiken and Vestergaard recently reviewed eleven prospective studies that assessed switching treatment after ALN or RIS in women with postmenopausal osteoporosis. All studies were of short duration (all 24 months or less) and none of the studies had the statistical power to assess fracture-reduction efficacy. ${ }^{17}$ Thus, they concluded that further long-term studies were needed, although transitioning to other therapies is a very frequent occurrence in clinical practice.

A

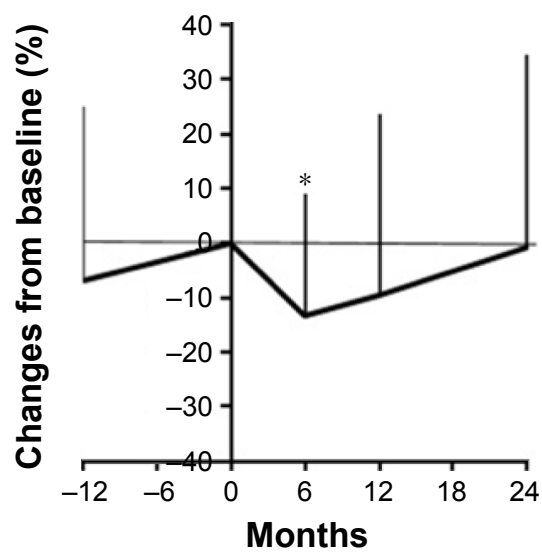

Among various bisphosphonates clinically available in Japan, we chose MIN in this study. MIN is reported to show the strongest inhibition of bone resorption among all the bisphosphonates used for osteoporosis treatment in Japan. ${ }^{7}$ Sakai et al recently reported that $50 \mathrm{mg}$ MIN monthly indeed alleviated low back pain, reduced bone turnover markers, increased bone density, and induced fewer upper gastrointestinal symptoms after switching from prior bisphosphonate treatment. ${ }^{18}$ More recently, switching weekly ALN or RIS to monthly $50 \mathrm{mg}$ MIN in patients with rheumatoid arthritis significantly increased lumbar and femoral neck BMDs at 12 months, indicating that switching to MIN may be an alternative treatment option. ${ }^{19}$ To the best of our knowledge, the present study is the first to evaluate the efficacy of switching therapy from RAL to MIN in patients with osteoporosis who no longer responded to RAL.

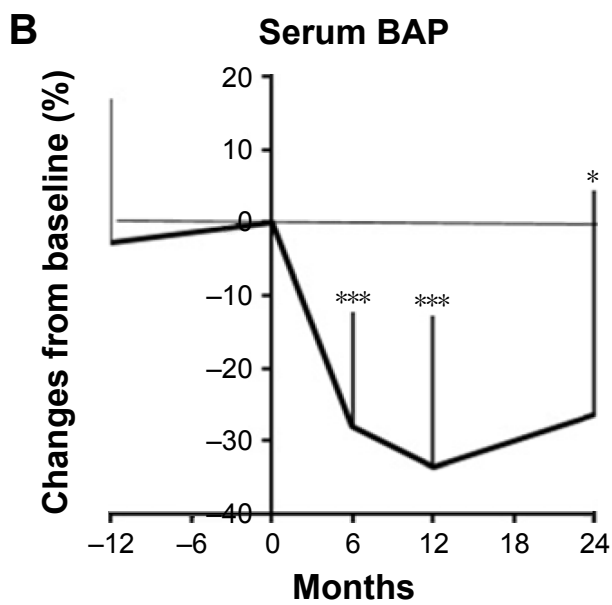

Figure 2 Percentage change of biochemical bone markers, serum NTX (A) and BAP (B) from the baseline during the follow-up period.

Notes: Points: mean, vertical lines: standard deviation, ${ }^{*} p<0.05$ and $* * * p<0.001$ versus baseline.

Abbreviations: NTX, Ncollagen-type N-telopeptide; BAP, bone-specific alkaline phosphatase. 


\section{Limitations}

This study has limitations. This is an open-label observational study involving a small number of patients, and patients are not randomized. It is pivotal to make an appropriate control to prove the efficacy, which we were unable to do. Further studies would be needed to confirm the findings in the present study.

\section{Conclusion}

Switching RAL to MIN significantly increased lumbar and femoral neck BMDs after 24 months, accompanied by the significant reduction of bone turnover marker, serum BAP, suggested that MIN is effective in RAL poor responders in clinical practice and should be considered as one of the treatment options for osteoporosis.

\section{Acknowledgments}

We thank Mami Morikawa for her secretarial help. This study was partly funded by Astellas Pharma Inc., (Tokyo, Japan).

\section{Disclosure}

The authors report no conflicts of interest in this work.

\section{References}

1. Das S, Crockett JC. Osteoporosis - a current view of pharmacological prevention and treatment. Drug Des Devel Ther. 2013;7:435-448.

2. Melton LJ 3rd, Khosla S, Malkasian GD, Achenbach SJ, Oberg AL, Riggs BL. Fracture risk after bilateral oophorectomy in elderly women. J Bone Miner Res. 2003;18(5):900-905.

3. Committee of Japanese Guidelines for the Prevention and Treatment of Osteoporosis. Japanese Guidelines for the Prevention and Treatment of Osteoporosis. Tokyo: Life Science; 2011.

4. Kanis JA, Johnell O, Black DM, et al. Effect of raloxifene on the risk of new vertebral fracture in postmenopausal women with osteopenia or osteoporosis: a reanalysis of the Multiple Outcomes of Raloxifene Evaluation trial. Bone. 2003;33(3):293-300.

5. Iikuni N, Hamaya E, Nihojima S, et al. Safety and effectiveness profile of raloxifene in long-term, prospective, postmarketing surveillance. $J$ Bone Miner Metab. 2012;30(6):674-682.
6. Dunford JE, Thompson K, Coxon FP, et al. Structure-activity relationships for inhibition of farnesyl diphosphate synthase in vitro and inhibition of bone resorption in vivo by nitrogen-containing bisphosphonates. J Pharmacol Exp Ther. 2001;296(2):235-242.

7. Tanishima S, Morio Y. A review of minodronic acid hydrate for the treatment of osteoporosis. Clin Interv Aging. 2013;8:185-189.

8. Matsumoto T, Hagino H, Shiraki M, et al. Effect of daily oral minodronate on vertebral fractures in Japanese postmenopausal women with established osteoporosis: a randomized placebo-controlled doubleblind study. Osteoporos Int. 2009;20(8):1429-1437.

9. Ettinger B, Black DM, Mitlak BH, et al. Reduction of vertebral fracture risk in postmenopausal women with osteoporosis treated with raloxifene: results from a 3-year randomized clinical trial. Multiple Outcomes of Raloxifene Evaluation (MORE) Investigators. JAMA. 1999;282(7):637-645.

10. Delmas PD, Ensrud KE, Adachi JD, et al. Efficacy of raloxifene on vertebral fracture risk reduction in postmenopausal women with osteoporosis: four-year results from a randomized clinical trial. J Clin Endocrinol Metab. 2002;87(8):3609-3617.

11. Qu Y, Wong M, Thiebaud D, Stock JL. The effect of raloxifene therapy on the risk of new clinical vertebral fractures at three and six months: a secondary analysis of the MORE trial. Curr Med Res Opin. 2005; 21(12):1955-1959.

12. Hagino H, Shiraki M, Fukunaga M, et al. Three years of treatment with minodronate in patients with postmenopausal osteoporosis. $J$ Bone Miner Metab. 2012;30(4):439-446.

13. Cosman F, Wermers RA, Recknor C, et al. Effects of teriparatide in postmenopausal women with osteoporosis on prior alendronate or raloxifene: differences between stopping and continuing the antiresorptive agent. J Clin Endocrinol Metab. 2009;94(10):3772-3780.

14. Cosman F, Keaveny TM, Kopperdahl D, et al. Hip and spine strength effects of adding versus switching to teriparatide in postmenopausal women with osteoporosis treated with prior alendronate or raloxifene. J Bone Miner Res. 2013;28(6):1328-1336.

15. Ebina K, Hashimoto J, Kashii M, et al. The effects of switching daily teriparatide to oral bisphosphonates or denosumab in patients with primary osteoporosis. J Bone Miner Metab. 2017;35(1):91-98.

16. Jakob F, Oertel H, Langdahl B, et al. Effects of teriparatide in postmenopausal women with osteoporosis pre-treated with bisphosphonates: 36-month results from the European Forsteo Observational Study. Eur J Endocrinol. 2012;166(1):87-97.

17. Eiken $P$, Vestergaard $P$. Treatment of osteoporosis after alendronate or risedronate. Osteoporos Int. 2016;27(1):1-12.

18. Sakai A, Ikeda S, Okimoto N, et al. Clinical efficacy and treatment persistence of monthly minodronate for osteoporotic patients unsatisfied with, and shifted from, daily or weekly bisphosphonates: the BP-MUSASHI study. Osteoporos Int. 2014;25(9):2245-2253.

19. Ebina K, Noguchi T, Hirao M, et al. Effects of switching weekly alendronate or risedronate to monthly minodronate in patients with rheumatoid arthritis: a 12-month prospective study. Osteoporos Int. 2016; 27(1):351-359.
International Journal of Women's Health

\section{Publish your work in this journal}

The International Journal of Women's Health is an international, peerreviewed open-access journal publishing original research, reports, editorials, reviews and commentaries on all aspects of women's healthcare including gynecology, obstetrics, and breast cancer. The manuscript management system is completely online and includes

\section{Dovepress}

a very quick and fair peer-review system, which is all easy to use. Visit http://www.dovepress.com/testimonials.php to read real quotes from published authors. 